walk test (ISW). However, these tests require space, repetition, and can be time-consuming. In the elderly population, gait speed alone has been shown to be a significant predictor of disability and mortality. We hypothesised that the 4-m gait speed in COPD patients would correlate well with the 6MW and ISW, and with validated COPD mortality composite scores such as BODE and ADO.

Methods 26 well-characterised COPD patients were studied. Each underwent $6 \mathrm{MW}$ test as per ATS guidelines, ISW test and completed a 4-m walk in random order. For the 4-m walk, participants were instructed to walk at their usual speed along a marked, flat unobstructed course. Timing was stopped when the first foot completely crossed the 4-m mark. The faster of two timed walks was used for scoring purposes, and a gait speed was calculated in $\mathrm{m} / \mathrm{s}$. Data were analysed using Spearman's rank correlation to assess association between 4-m gait speed and 6MW, ISW, BODE score and $\mathrm{ADO}$ index.

Results Baseline characteristics are presented as mean (SD) or median (25th, 75th percentile): 11M: $15 \mathrm{~F}$; age $=69$ (8); FEV1\% predicted $=43$ (20); $6 \mathrm{MW}=330$ (83); ISW=291 (122.5); MRC dyspnoea 3 (2, 4); St George's Respiratory Questionnaire (SGRQ)= 49.6 (21.0); $\mathrm{BMI}=25.9$ (4.9); $\mathrm{BODE}=4.5(1.75,6.0), \mathrm{ADO}=5(4,6)$. Mean (SD) 4-m gait speed was $0.96(0.20) \mathrm{m} / \mathrm{s}$. There was a significant correlation between $4-\mathrm{m}$ gait speed and $6 \mathrm{MW}(\mathrm{r}=0.62$, $\mathrm{p}=<0.001)$, ISW $(\mathrm{r}=0.80, \mathrm{p}=<0.0001)$-see Abstract S73 Figure 1, BODE score $(r=-0.56, p=0.003)$ and $\mathrm{ADO}$ index $(\mathrm{r}=-0.43$, $\mathrm{p}=0.03)$.

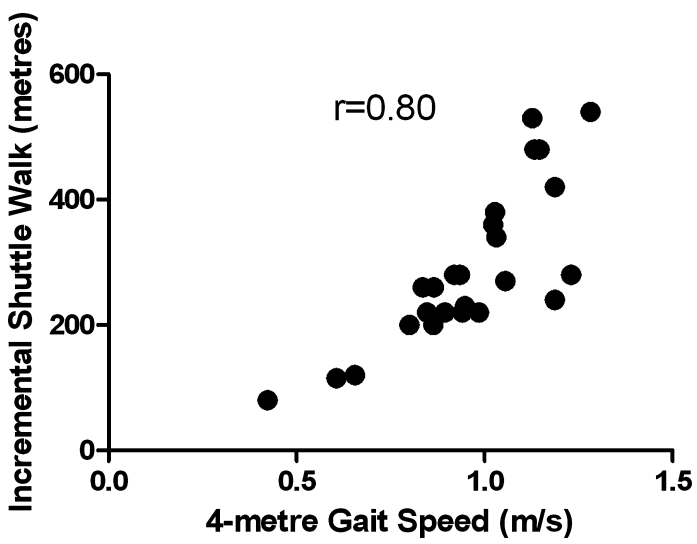

Abstract S73 Figure 1

Conclusions 4-m gait speed correlates well with existing functional outcome measures and validated mortality composite scores used in COPD. It is an easy and quick to perform field test, and does not require specialist training or equipment. Further longitudinal and intervention studies are required to validate the 4-m gait speed as an assessment tool.

\section{S74 EFFECT OF PULMONARY REHABILITATION ON THE SHORT PHYSICAL PERFORMANCE BATTERY (SPPB) IN COPD}

\section{doi:10.1136/thx.2010.150938.25}

M S Patel, A L Clark, K A Ingram, R P Fowler, A V Donaldson, S S Kon, M I Polkey, W D Man. Respiratory Biomedical Research Unit and Harefield Pulmonary Rehabilitation Team, Royal Brompton \& Harefield NHS Foundation Trust, UK

Introduction The Short Physical Performance Battery (SPPB) is a simple objective assessment tool developed by the National Institute on Ageing for evaluating lower extremity function in older persons. It comprises tests of standing balance, normal gait speed and timed sit-to-stand with a maximum score of 12, and predicts nursing home admission rates and mortality. Lower extremity dysfunction is well recognised in COPD, and hence the SPPB may be a useful functional outcome measure in COPD patients. Pulmonary rehabilitation (PR) is a highly effective intervention in COPD and ameliorates lower limb dysfunction. We hypothesised that the SPPB would improve after PR.

Methods 35 COPD (18M:17F) patients underwent an 8 week outpatient pulmonary rehabilitation programme. The SPPB, incremental shuttle walk (ISW) and St George's Respiratory Questionnaire (SGRQ) were measured immediately before and after a PR programme. Paired $t$-test (or non-parametric equivalent) was used to test the effect of PR.

Results Baseline characteristics are expressed as mean (SD) or median (25th, 75th percentiles): age 69 (8), FEV1\% 43 (23), MRC Dyspnoea 4 (3, 4), ISW 130 (60, 270), and SGRO 57 (16). Following $\mathrm{PR}$, there was a significant improvement in ISW (mean $70 \mathrm{~m}$; $\mathrm{p}<0.001)$ and SGRQ $(-7.0 ; \mathrm{p}<0.005)$. SPPB also significantly improved from a median of $9(6,11)$ pre-PR to a median of $11(7,12$; $\mathrm{p}<0.004)$ post-PR. Change in SPPB did not correlate with change in ISW $(r=0.22 ; \mathrm{p}=0.20)$.

Conclusions The SPPB may be a useful functional outcome measure in COPD, and gives different information from the ISW. Larger studies are required to determine the minimum clinically significant change in the SPPB.

Contributors M S Patel, A L Clark contributed equally to this study.

\section{S75 IS A PRACTICE INCREMENTAL SHUTTLE WALK TEST ALWAYS NECESSARY AND IS IT INFLUENCED BY MRC DYSPNOEA GRADE?}

doi:10.1136/thx.2010.150938.26

${ }^{1} \mathrm{~A}$ K Dhaliwal, ${ }^{1} \mathrm{~L}$ Sewell, ${ }^{1} \mathrm{R}$ Collier, ${ }^{1,2} \mathrm{~S} \mathrm{~J}$ Singh. ${ }^{1}$ Department of pulmonary rehabilitation, Glenfield hospital, University Hospitals of Leicester, Leicester, UK; ${ }^{2}$ Coventry University, Coventry, UK

Introduction The Incremental Shuttle Walk Test (ISWT) is a common assessment tool used to assess exercise capacity in patients with Chronic Obstructive Pulmonary Disease (COPD). Healthcare professionals conducting this test will ask patients to perform a practice Incremental Shuttle Walk Test (PISWT). The aim was to explore whether a PISWT is needed and to examine whether variance between PISWT and the second shuttle walk test (ISWT2) is influenced by MRC dyspnoea grade.

Method This is a retrospective audit of 441 COPD patients: male 194 female 297 mean (SD) age 68.9 (10.13) years who were assessed for outpatient pulmonary rehabilitation (PR). Patients undertook a PISWT and rested for 30 min before performing ISWT2. Distance covered (m), MRC grade, heart rate (HR), forced expiratory volume in $1 \mathrm{~s}$ (FEV1), forced vital capacity (FVC) and body mass index (BMI) were recorded.

Results The mean (SD) change between both shuttle walk tests and significance levels were analysed using a paired t test. These results are detailed in Abstract S75 Table 1. Overall, there was a statistically significant increase in mean walking distance between PISWT and ISWT2. In each patient subgroup, a significant increase was seen for all, except for those of MRC grade 1; however this group consisted only of eight patients. Furthermore, no patient subgroup achieved the minimum clinically important distance for the ISWT $>47.5 \mathrm{~m}$ (Singh et al, 2008). Patients in MRC grades 2 and 3 showed more variance between each shuttle walk test. The least mean change was seen in patients of MRC grade 5 . 
Abstract S75 Table 1

\begin{tabular}{|c|c|c|c|c|c|c|}
\hline $\begin{array}{l}\text { MRC } \\
\text { Grade }\end{array}$ & $\begin{array}{l}\text { Mean change } \\
\text { (SD) ISWT (m) }\end{array}$ & ) $95 \%$ C.I & $\begin{array}{l}\text { Sig. } \\
\text { (2 tailed) }\end{array}$ & $\begin{array}{l}\text { FEV1 } \\
\text { (litres) (SD) }\end{array}$ & $\begin{array}{l}\text { FVC } \\
\text { (litres) (SD) }\end{array}$ & ) BMI (SD) \\
\hline $1 \mathrm{n}=8$ & $11.25(45.49)$ & -26.78 to 49.28 & 507 & $1.38(0.41)$ & $2.39(0.57)$ & $27.90(6.05)$ \\
\hline $2 n=88$ & $29.54(46.11)$ & 19.77 to 39.31 & $<0.0001$ & $1.43(0.57)$ & $2.62(0.67)$ & $26.30(5.50)$ \\
\hline $3 n=142$ & 223.88 (44.98) & 15.91 to 30.84 & $<0.0001$ & $1.39(0.56)$ & $2.46(0.74)$ & $28.60(5.90)$ \\
\hline $4 n=128$ & 814.37 (34.70) & 8.30 to 20.44 & $<0.0001$ & $1.15(0.55)$ & $2.34(0.94)$ & 27.61 (6.69) \\
\hline $5 n=75$ & $8.54(29.75)$ & 1.70 to 15.39 & 0.015 & $0.99(0.44)$ & $2.08(0.58)$ & $27.90(7.83)$ \\
\hline $\begin{array}{l}\text { Overall } \\
\text { change } \\
n=441\end{array}$ & $19.25(40.65)$ & 15.44 to 23.05 & $<0.0001$ & 10 & 0 & 0 \\
\hline
\end{tabular}

Conclusion Overall, there was a statistical significant increase in distance walked in all patients. These data confirm the need for a PISWT to be conducted within the COPD population. Patients in MRC grades 2 and 3 show a larger variance in walking distance between both shuttle walk tests.

\section{S76 THE COPD ASSESSMENT TEST SCORE (CAT): A MULTICENTRE, PROSPECTIVE STUDY OF RESPONSE TO PULMONARY REHABILITATION}

doi:10.1136/thx.2010.150938.27

${ }^{1} \mathrm{~J}$ W Dodd, ${ }^{2} \mathrm{~L}$ Hogg, ${ }^{1} \mathrm{~J}$ Nolan, ${ }^{3} \mathrm{H}$ Jefford, ${ }^{4} \mathrm{~V} M$ Lord, ${ }^{5} \mathrm{R}$ Garrod, ${ }^{4} \mathrm{M}$ I Polkey, ${ }^{1} \mathrm{P}$ W Jones, ${ }^{4}$ W D-C Man, ${ }^{4} \mathrm{~N}$ S Hopkinson. ${ }^{1}$ St George's Hospital, London, UK; ${ }^{2}$ St Thomas' Hospital, London, UK; ${ }^{3}$ Greenwich PCT, London, UK; ${ }^{4} \mathrm{NHLI}$, Imperial College, Royal Brompton Hospital, London, UK; ${ }^{5}$ King's College Hospital, London, UK

Background The COPD assessment test (CAT) was introduced last year. ${ }^{1}$ It is a questionnaire that contains eight questions covering domains relating to the impact of COPD symptoms. It is a self completion questionnaire and does not require scoring software. Scores of $0-10,11-20,21-30,31-40$ represent mild, moderate, severe or very severe clinical impact. In cross-sectional studies it has similar scaling properties to the SGRQ, so that 1 point in the CAT is equivalent to approximately 2.5 points on the SGRO. It is not known how the CAT score performs in the context of pulmonary rehabilitation (PR)

Methods We prospectively studied the introduction of the CAT score as an assessment tool in several pulmonary rehabilitation programs across London, where it was used alongside a range of other outcome measures in different programs including the SGRQ, CCO, $\mathrm{HAD}$ score, MRC dyspnoea score and several different walking tests. Primary outcome was a comparison of change in CAT score against an anchor question used to assess overall response, scored 1 "I feel much better" 2 "I feel a little better", 3 "I feel no different", 4 "I feel a little worse", 5 "I feel much worse".

Results Data were available for 172 COPD patients, mean(SD) age 69.6(9.3) years, $\mathrm{FEV}_{1}$ 51.9(18.9)\% predicted, MRC dyspnoea score 3.0(0.9), CAT score 20.0(7.5) who attended five different programs. Mean improvement in CAT score after PR was 2.8(5.8) points. In those scoring "much better" $(\mathrm{n}=108)$ CAT fell by 3.7(6.1) points and by $1.2(4.8)$ in those who felt "a little better" $(n=56)(p=0.01)$. In those scoring 3 or 4 on the anchor question (grouped together as $\mathrm{n}=8$ ) change in CAT was -0.6(3.5).

Conclusion The CAT score improves in response to pulmonary rehabilitation and more so in those who report a greater overall improvement. Further accrual is underway to allow comparison of changes in CAT to other outcome measures.

Funding The NIHR Respiratory Biomedical Research Unit Royal Brompton \& Harefield NHS Foundation Trust and Imperial College.

\section{REFERENCE}

1. Jones PW. Eur Respir J 2009; 34:648-54.
Mechanisms of lung infection in the community and hospital setting

S77 COMPARTMENTALISATION OF SURFACE TRIGGERING
RECEPTOR EXPRESSED ON MYELOID CELLS-1 (TREM-1) IN
VENTILATOR-ASSOCIATED PNEUMONIA (VAP)

doi:10.1136/thx.2010.150938.28

V Grover, P Kelleher, D Henderson, N Soni, S Singh. Chelsea and Westminster NHS Foundation Trust, London, UK

Introduction Biomarkers have been investigated in order to speed up diagnosis of VAP, a common condition in ICU patients. TREM-1 is a protein involved in amplification of immune responses to bacterial and fungal infection and exists as soluble and surface forms. ${ }^{12}$ The diagnostic value of soluble TREM-1 in broncho-alveolar lavage fluid (BALF) in VAP is controversial. ${ }^{3}$ Therefore the utility of surface TREM-1 for diagnosing VAP in a two-compartment model (BALF and blood) was investigated.

Methodology Paired blood and BALF were obtained in consenting patients in the following groups: (1) Ventilated patients with VAP diagnosed on semi-quantitative microbiology and Clinical Pulmonary Infection Score (CPIS); (2) Ventilated patients without sepsis; (3) Day-case bronchoscopy patients without evidence of infection. Flow cytometry was performed on cell pellets derived from simultaneous BALF and blood samples. Surface TREM-1, CD11b (immune cell activation marker) and L-selectin (immune cell migration marker) levels were measured on monocytes and neutrophils. At the same time an inflammatory cytokine panel (comprising IL-1 $\beta$, IL-6, IL-8 and soluble TREM-1) was measured by ELISA in the paired blood and BALF samples.

Results Expression of TREM-1 and CD11b on monocytes were significantly elevated in BALF samples obtained from the VAP patient group. There was no change in blood surface TREM-1 and CD11b levels between the different patient groups. The BALF/blood ratio of monocytic TREM-1 increased the discrimination between

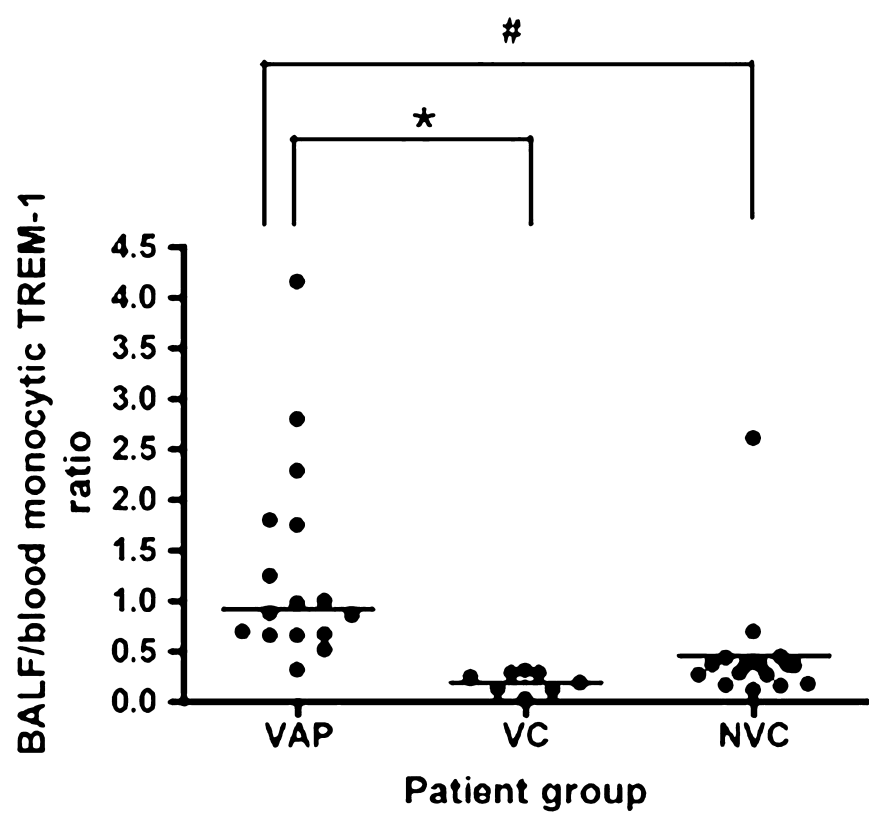

Abstract S77 Figure 1 The ratio monocytic surface TREM-1 between BALF and blood (flow cytometry). 16 patients with VAP are compared with 8 ventilated non-septic control (VC) and 17 non-ventilated noninfected control patients (NVC). The median levels and IQRs are: VAP $(0.92,0.66-1.77)$, VC $(0.21,0.12-0.28)$ and NVC $(0.36,0.22-0.41)$. $^{*}$ and \# $\mathrm{p}=0.0001$. 